\title{
INSTABILITIES OF THE GINZBURG-LANDAU EQUATION: PERIODIC SOLUTIONS*
}

\author{
BY \\ PAUL K. NEWTON ${ }^{1}$ AND LAWRENCE SIROVICH \\ Brown University
}

\begin{abstract}
The evolution of spatially periodic unstable solutions to the Ginzburg-Landau equation is considered. These solutions are shown to remain pointwise bounded (Lagrange stable). The first step in the route to chaos is limit cycle behavior. This is treated by perturbation theory and shown to result in a factorable form. Agreement between the perturbation result and an exact numerical integration is shown to be excellent.
\end{abstract}

1. Introduction. Recent numerical studies on transition to chaos have focused on the Ginzburg-Landau equation $[1,2,3,4,5,6,7]$,

$$
A_{t}-\left(\lambda_{r}+i \lambda_{i}\right) A_{x x}=\sigma_{r} A-\left(\beta_{r}+i \beta_{i}\right)|A|^{2} A .
$$

Here $\lambda_{r}, \lambda_{i}, \sigma_{r}, \beta_{r}, \beta_{i}$ represent real quantities. This equation has been derived as a wave envelope or amplitude equation governing wave-packet solutions in a variety of fluid stability problems for which an external parameter is slightly supercritical $[8,9,10,11,12]$. Under the limit $\sigma_{r}=0, \lambda_{r}=0, \lambda_{i}=1, \beta_{r}=0$, this reduces to the cubic Schrödinger equation of deep water gravity waves, which has also been derived as a far field approximation for dispersive systems describing the amplitude of quasimonochromatic waves in a wide range of physical problems $[13,14,15,16]$.

As is easily seen, (1) possesses a uniform in space, sinusoidal in time solution known as the Stokes solution. Elementary considerations show this to be unstable to periodic disturbances, a fact directly related to the well-known sideband instability [13, 17, 18]. As the numerical studies show, this leads, with decreasing wave number, to chaos. Nevertheless as shown in Sec. 3 growing solutions remain pointwise bounded. Both the numerical investigations (see especially $[1,2,4,6]$ ) and the analytical treatment given in Sec. 4 demonstrate that the first step in the route to chaos is a limit cycle. Although this is shown by perturbation theory, an exact consequence is that limit cycle behavior is described by a

\footnotetext{
${ }^{*}$ Received November 26, 1984.

' Present address: Dept. of Math. Stanford Univ. Stanford, CA 94305
} 
factorable solution to (1). Finally in Sec. 5 we show that the perturbation solution is in excellent agreement with the exact solution (numerical) well beyond the range of the small parameter of the problem.

2. Normalization and linear stability. If (1) is scaled by

$$
t^{\prime}=\lambda_{i}\left|\sigma_{r} / \lambda_{r}\right| t, \quad x^{\prime}=\left|\sigma_{r} / \lambda_{r}\right|^{1 / 2} x, \quad A^{\prime}=\left|\beta_{r} / \sigma_{r}\right|^{1 / 2} A
$$

we obtain

$$
i A_{t^{\prime}}^{\prime}+\left(1-i c_{0}\right) A_{x^{\prime} x^{\prime}}^{\prime}=i c_{0} A^{\prime}-\left(c_{1}+i c_{0}\right)\left|A^{\prime}\right|^{2} A^{\prime}
$$

with

$$
c_{0}=\left|\lambda_{r}\right| / \lambda_{i}, \quad c_{1}=-\beta_{i}\left|\lambda_{r}\right| /\left|\beta_{r}\right| \lambda_{i} .
$$

For simplicity we take $\lambda_{r}, \sigma_{r}, \beta_{r}, \lambda_{i},-\beta_{i} \geqslant 0$. An alternative useful scaling is

$$
t^{\prime \prime}=c_{1} t^{\prime}, \quad X^{\prime \prime}=X^{\prime} \sqrt{c_{1}}
$$

in which case (3) becomes

$$
i A_{t}+\left(1-i c_{0}\right) A_{x x}=i \frac{c_{0}}{c_{1}} A-\left(1+i \frac{c_{0}}{c_{1}}\right)|A|^{2} A,
$$

where all primes have been dropped.

Equation (6) is satisfied by the Stokes solution:

$$
A=\exp (i t) \text {. }
$$

If the Stokes solution (7) is perturbed by a spatially periodic disturbance of wavelength $L=2 \pi / q$, it then follows that (7) is linearly stable unless

$$
q^{2}<\frac{2\left(1-c_{0}^{2} / c_{1}\right)}{\left(1+c_{0}^{2}\right)}=q_{0}^{2} .
$$

(Typical plots of $q_{0}$ are shown in Fig. 1.) In fact, at least $n$ harmonics are unstable if

$$
q^{2}<\frac{2\left(1-c_{0}^{2} / c_{1}\right)}{n^{2}\left(1+c_{0}^{2}\right)} .
$$

For small departures from the neutral curve of size

$$
\varepsilon^{2}=q_{0}^{2}-q^{2},
$$

the temporal growth rate is easily seen to be $O\left(\varepsilon^{2}\right)$.

3. Lagrange stability. In view of the instability of (7) to periodic disturbances we now examine the consequences of temporal growth. Our discussion is based on that given by Thyagaraja [20] for the cubic Schrödinger equation.

To be specific we introduce the energy

$$
I=\int_{0}^{L}|A(x, t)|^{2} d x=\|A\|_{2}^{2}
$$


and consider the case of growth,

$$
\frac{\partial I}{\partial t} \geqslant 0
$$

The energy form of (6) is obtained by multiplying it by the conjugate $A^{*}$, subtracting the conjugate of the result and then integrating over the period, $L$. This yields

$$
\frac{\partial I}{\partial t}=-2 c_{0} Q I+\frac{2 c_{0}}{c_{1}} I-\frac{2 c_{0}}{c_{1}} \int_{0}^{L}|A|^{4} d x
$$

where

$$
Q=\int_{0}^{L}\left|A_{x}\right|^{2} d x / I
$$

is the Rayleigh quotient. For later purposes we point out that the last term of (13), by the Schwarz inequality is

$$
\int_{0}^{L}|A|^{4} d x \geqslant I^{2} / L
$$

Through a series of lemmas we will prove that if (12) holds,

$$
\|A\|_{\infty}^{2}=\max _{0 \leqslant x \leqslant L}|A(x, t)|^{2}<K
$$

for all $t \geqslant 0$ where $K$ is constant. If (16) holds, a solution is said to be Lagrange stable [21].

We start the demonstration with

LEMMA 1 . In the parameter region where (12) holds,

$$
I(t) \leqslant L
$$

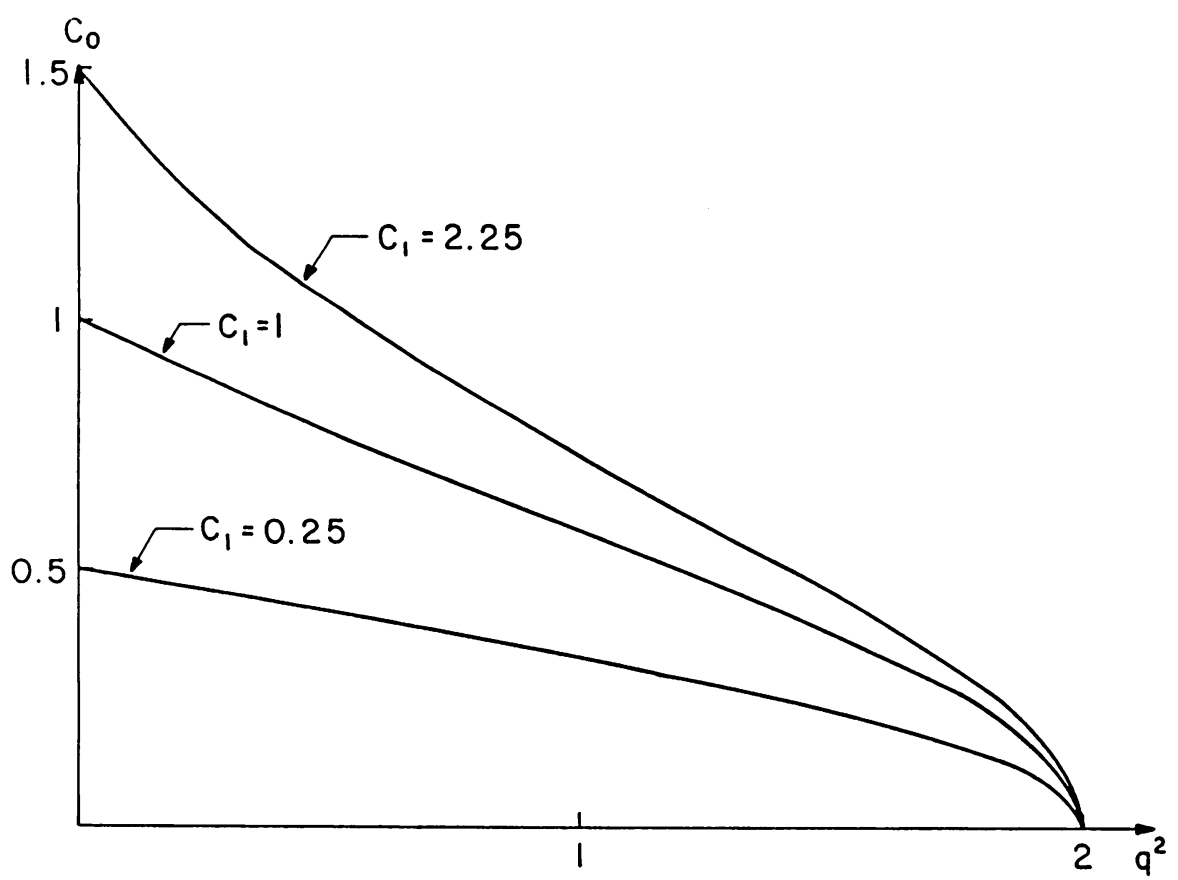

FIG. 1. First transition curve $q^{2}=q_{0}^{2}$ (see Eq. (8)) for the cases $c_{1}=2.25, c_{1}=1, c_{1}=.25$. 
and

$$
\frac{\partial I}{\partial t} \leqslant \frac{L c_{0}}{2 c_{1}} .
$$

Proof. From (13) and the fact that $I$ and $Q$ are both nonnegative,

$$
\frac{\partial I}{\partial t} \leqslant \frac{2 c_{0}}{c_{1}} I-\frac{2 c_{0}}{c_{1}} \int_{0}^{L}|A|^{4} d x .
$$

Thus from (15),

$$
\frac{\partial I}{\partial t} \leqslant I\left[\frac{2 c_{0}}{c_{1}}-\frac{2 c_{0}}{c_{1} L} I\right],
$$

and (18) follows directly. Next with the assumption $\partial I / \partial t \geqslant 0$ we have

$$
I\left[\frac{2 c_{0}}{c_{1}}-\frac{2 c_{0}}{c_{1} L} I\right] \geqslant 0
$$

which gives (17).

Lemma 2. For the parameter region in which (12) holds, the Rayleigh quotient has the bound

$$
Q(t) \leqslant 1 / c_{1}
$$

and further

$$
\max _{0 \leqslant x \leqslant L}|A(x, t)|^{2} \leqslant L\left(\frac{1}{L}+\frac{2}{\sqrt{c_{1}}}\right) \text { for } t \geqslant 0 .
$$

Proof. At any instant of time, let $x_{0}$ be a point at which $|A(x, t)|^{2}$ takes its global minimum value. Consider:

$$
A^{2}(x, t)=A^{2}\left(x_{0}, t\right)+2 \int_{x_{0}}^{x} A A_{x} d x
$$

and from it

$$
|A(x, t)|^{2} \leqslant\left|A\left(x_{0}, t\right)\right|^{2}+2 \int_{0}^{L}\left|A A_{x}\right| d x .
$$

Next observe

$$
\left|A\left(x_{0}, t\right)\right|^{2}=\frac{1}{L} \int_{0}^{L}\left|A\left(x_{0}, t\right)\right|^{2} d x \leqslant \frac{1}{L} \int_{0}^{L}|A(x, t)|^{2} d x
$$

and from the Cauchy-Schwarz inequality we have

$$
\int_{0}^{L}\left|A A_{x}\right| d x \leqslant I Q^{1 / 2}
$$

Introducing these relations into (25) yields

$$
|A(x, t)|^{2} \leqslant I\left(\frac{1}{L}+2 Q^{1 / 2}\right) .
$$

If we return to (13),

$$
Q=\frac{1}{I}\left\{-\frac{1}{2 c_{0}} \frac{\partial I}{\partial t}+\frac{I}{c_{1}}-\frac{1}{c_{1}} \int_{0}^{L}|A|^{4} d x\right\}
$$


we may now state,

$$
Q \leqslant 1 / c_{1}
$$

Thus from (30) and (17), (28) gives

$$
|A(x, t)|^{2} \leqslant L\left(\frac{1}{L}+2 / \sqrt{c_{1}}\right),
$$

and Lagrange stability is established. Thus pointwise boundedness is obtained even when the overall energy shows growth.

4. Limit cycle solutions. The boundedness just demonstrated and the numerical studies mentioned in the introduction suggest that the Stokes solution first bifurcates to a limit cycle. To examine this assertion, we might approach the problem through a perturbation analysis about the Stokes solution. To avoid non-uniformities we remark that the proposed solution, will in general have a new period. To accommodate this effect we write the perturbed form of the solution as

$$
A(x, t: \varepsilon)=(1+\phi(x, t: \varepsilon)) \exp (i \Omega(\varepsilon) t),
$$

where $\Omega(0)=1$ ( $\varepsilon$ is defined by (10)). Time is rescaled by

$$
\tau=\Omega t
$$

which with (32) transforms (6) to

$$
i \Omega \phi_{\tau}+\left(1-i c_{0}\right) \phi_{x x}=(\Omega-1)(1+\phi)-\left(1+i \frac{c_{0}}{c_{1}}\right)\left[\phi+\phi^{*}+\phi^{2}+2 \phi \phi^{*}+\phi^{2} \phi^{*}\right] .
$$

Since (34) supports an even solution, we consider

$$
\phi=\sum_{n=0} a_{n}(\tau ; \varepsilon) \cos (n q x),
$$

where $a_{n}=\alpha_{n}+i \beta_{n}$ are functions of time and $\varepsilon$. A study of the perturbation analysis soon shows that we may adopt the following 'Ansatz':

$$
\begin{gathered}
\Omega=1+\sum_{n=1} \Omega_{2 n} \varepsilon^{2 n}, \\
a_{0}=\sum_{n=1} a_{2 n}^{(0)} \varepsilon^{2 n}, \\
a_{k}=\sum_{n=0} a_{2 n+k}^{(k)} \varepsilon^{2 n+k}, \quad k>0 .
\end{gathered}
$$

Insertion of these forms into (34) results in a straightforward perturbation analysis and we avoid the details. Since we are primarily interested in limit cycle solutions, we simplify our analysis if we drop transients. Thus we immediately look for periodic solutions to $a_{k}(\tau)$. If this is carried out it has the consequence that

$$
\phi_{\tau}=0,
$$

i.e., the time behavior is factorable in the form given by (32). 
The remainder of the perturbation is quite routine and the results to $O\left(\varepsilon^{2}\right)$ are given below:

$$
\left(\alpha_{1}^{(1)}\right)^{2}=N / D
$$

where

$$
\begin{aligned}
N= & -6 c_{0}^{8} c_{1}-6 c_{0}^{6} c_{1}\left(1-3 c_{1}\right)+18 c_{0}^{4} c_{1}^{2}\left(1-c_{1}\right)-6 c_{0}^{2} c_{1}^{3}\left(3-c_{1}\right)+6 c_{1}^{4}, \\
D= & 18 c_{0}^{8}-3 c_{0}^{6}\left(7 c_{1}^{2}+34 c_{1}+3\right) \\
& +c_{0}^{4}\left(c_{1}^{4}+58 c_{1}^{3}+186 c_{1}^{2}+22 c_{1}+1\right) \\
& -9 c_{0}^{2} c_{1}^{2}\left(5 c_{1}^{2}+14 c_{1}+1\right)+6 c_{1}^{4}\left(2 c_{1}+5\right), \\
\beta_{1}^{(1)}= & c_{0}\left(1+c_{1}\right) \alpha_{1}^{(1)} /\left(c_{1}-c_{0}^{2}\right), \\
\alpha_{2}^{(0)}= & \left(-5 c_{1}^{2}+6 c_{0}^{2} c_{1}+c_{0}^{2} c_{1}^{2}-2 c_{1}^{3}\right. \\
& \left.-3 c_{0}^{4}-c_{0}^{2}\right)\left(\alpha_{1}^{(1)}\right)^{2} / 4\left(c_{1}-c_{0}^{2}\right)^{2}, \\
\beta_{2}^{(0)}= & 0 \\
\Omega_{2}= & \left(-2 c_{1}^{3}+2 c_{0}^{2} c_{1}^{3}-2 c_{1}^{4}+2 c_{0}^{4} c_{1}\right. \\
& \left.-2 c_{0}^{2} c_{1}+2 c_{0}^{4}\right)\left(\alpha_{1}^{(1)}\right)^{2} / 2 c_{1}\left(c_{1}-c_{0}^{2}\right)^{2}, \\
\alpha_{2}^{(2)}= & \frac{1}{4}\left(\alpha_{1}^{(1)}\right)^{2}\left[1-\frac{c_{0}^{2}\left(1+c_{1}\right)^{2}}{3\left(c_{1}-c_{0}^{2}\right)^{2}}\right], \\
\beta_{2}^{(2)}= & \frac{1}{8}\left(\alpha_{1}^{(1)}\right)^{2}\left[\frac{3 c_{0}\left(1+c_{1}\right)}{\left(c_{1}-c_{0}^{2}\right)}+\frac{c_{0}^{3}\left(1+c_{1}\right)^{3}}{3\left(c_{1}-c_{0}^{2}\right)^{3}}\right] .
\end{aligned}
$$

The computations have been carried out to $O\left(\varepsilon^{4}\right)$; however, in view of the length of these expressions we only show the above. The algebra leading to (40)-(48) has in all instances been verified with the use of Macsyma.

5. Comparison with exact solutions. For limit cycle solutions of (6), the spatial part satisfies

$$
\left(1-i c_{0}\right) \frac{d^{2} F}{d x^{2}}=\left(\Omega+i \frac{c_{0}}{c_{1}}\right) F-\left(1+i \frac{c_{0}}{c_{1}}\right)|F|^{2} F
$$

where $F=(1+\phi)$. Since $F$ is complex, (49) represents a pair of coupled second-order equations or equivalently, a fourth-order equation. As is easily verified, (49) is invariant under the group $F \rightarrow F \exp (i \theta)$ and hence (49) may be reduced to a third-order equation. We have used this to calculate exact numerical solutions, which we now compare with the perturbation solutions discussed in the previous section. The comparisons are shown in Figs. 2, 3, 4, in which we display the numerical solution (dashed line) and the perturbation solution (solid line) at parameter values $c_{0}=.25, c_{1}=1$ (this corresponds to the calculations of Refs. $1,2, \& 6$ ), and for the cases $\Omega=.9, .83, .7$. The small parameter, $\varepsilon$, for each 

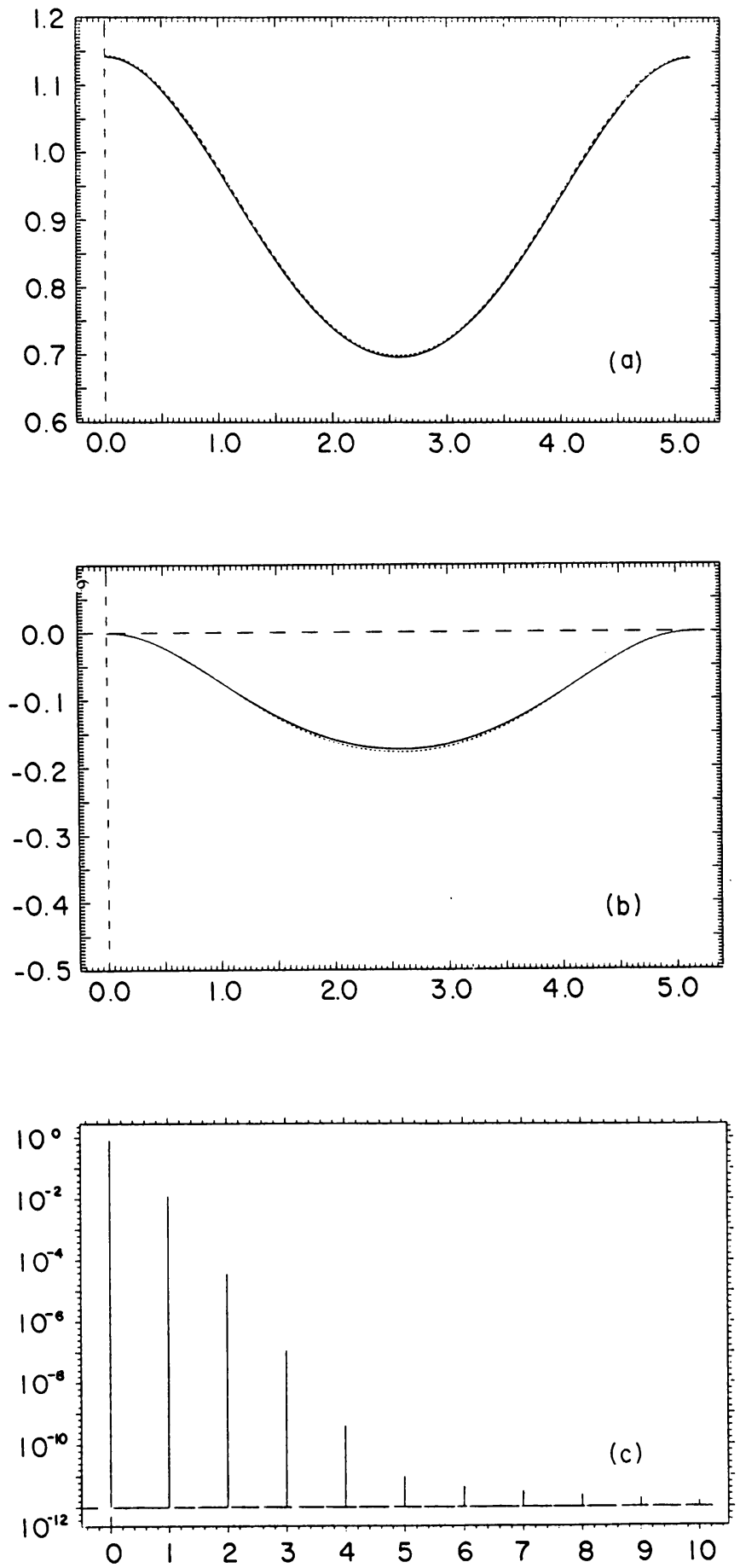

FIG. 2. Periodic solutions for parameter values $c_{0}=.25, c_{1}=1, \Omega=.9, \varepsilon=.52, q=1.22$. A: Comparison of real parts (dashed line is the numerical solution, solid line the perturbation solution). B: Comparison of imaginary parts. C: Power spectrum of the numerical solution with ordinate marked in inverse powers of 10 starting at 0 . 

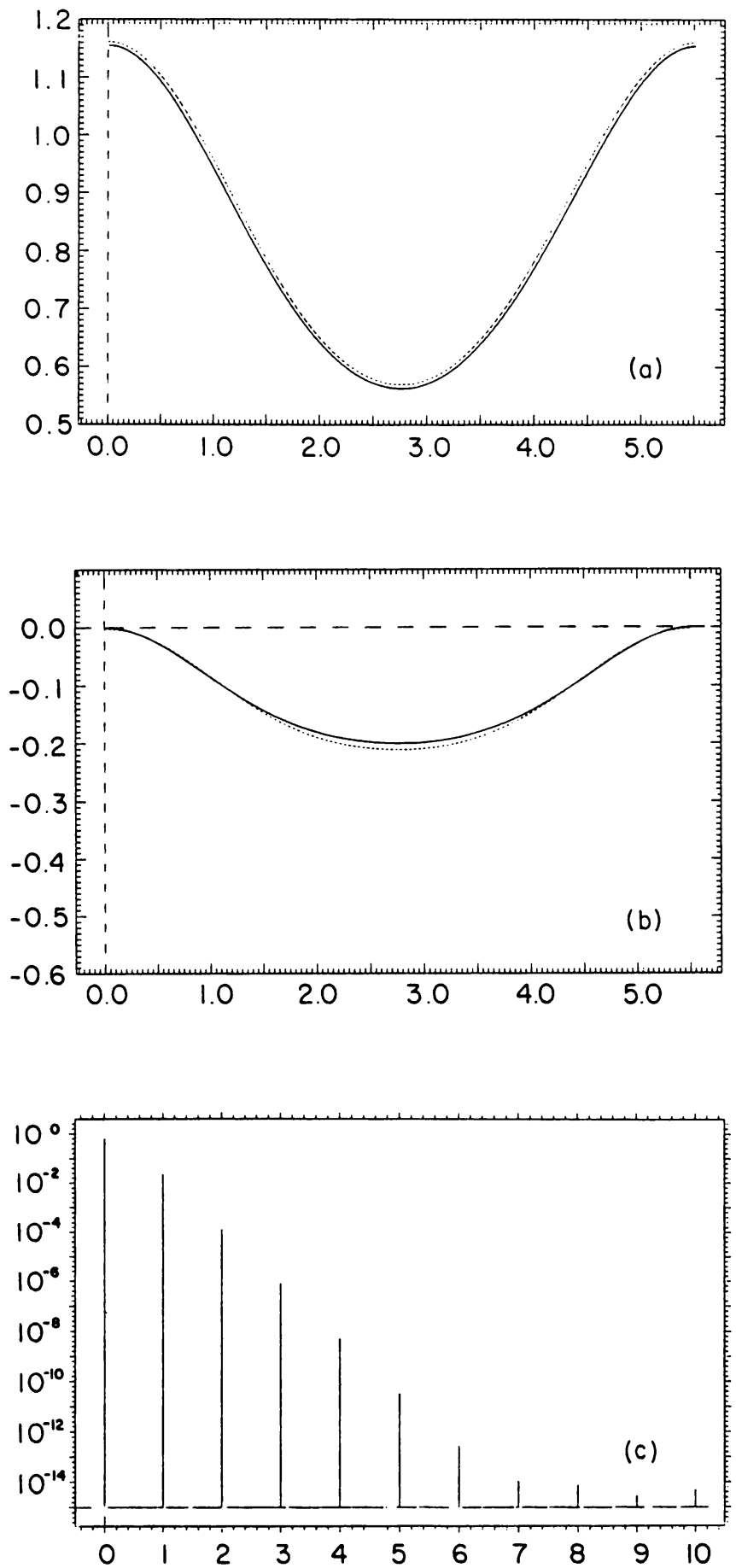

FIG. 3. Periodic solutions for parameter values $c_{0}=.25, c_{1}=1, \Omega=.83, \varepsilon=.68, q=1.14$. See Fig. 2 for rest. 

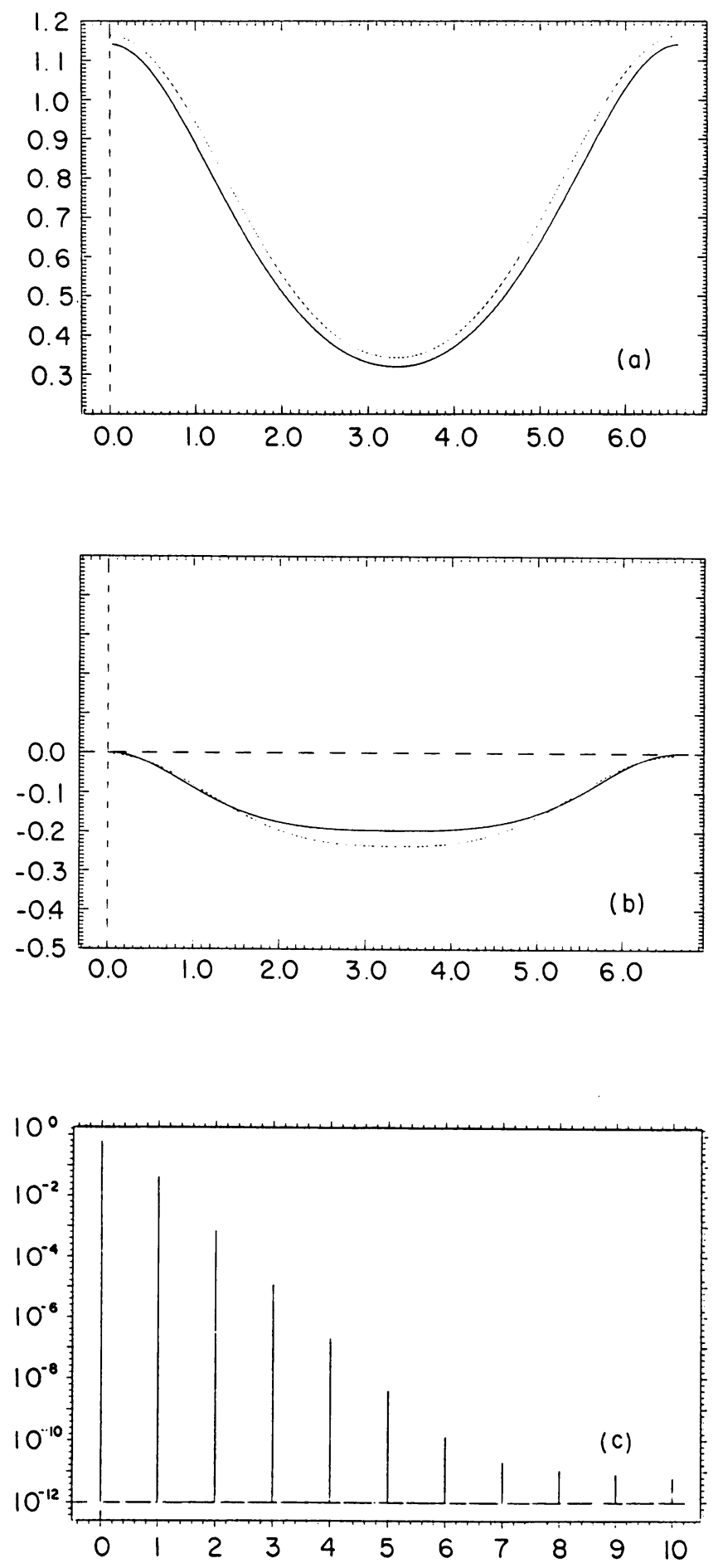

FIG. 4. Periodic solutions for parameter values $c_{0}=.25, c_{1}=1, \Omega=.7, \varepsilon=.93, q=.95$. See Fig. 2 for rest. 
of these cases is $.52, .68$, and .93 respectively. The power spectrum of the numerical solution is also displayed. The corresponding wavenumbers are given in the figure captions. The root mean square error averaged over the period between the perturbation solution and the exact solution for the three cases is . $5 \%, 2 \%, 6 \%$ respectively.

The good agreement between perturbation and exact theory is remarkable especially since $\varepsilon$ is quite large. (For cases not shown, comparable good agreement was found for cases in which $\varepsilon>1$.) A check of this phenomena shows that it is due to numerical smallness in the coefficients of (35), as might be expected.

Another feature to note in Figs. 2, 3, 4 is the linear fall off of the principal amplitudes in the energy spectrum. This is clearly in agreement with the perturbation analysis. Up to the order considered in the perturbation theory of Sec. 4, only $\left|a_{k}\right|^{2}, k=0, \ldots, 4$, contribute to the energy spectrum. In each case shown, these show good agreement with the displayed exact results.

\section{REFERENCES}

[1] H. T. Moon, P. Huerre, L. G. Redekopp, Three-frequency motion and chaos in the Ginzburg-Landau equation, Phys. Rev. Letters, Vol. 49, \# 7, 16 Aug. 1982

[2] H. T. Moon, P. Huerre, L. G. Redekopp, Transitions to chaos in the Ginzburg-Landau equation, Physica 7D, 135 (1983)

[3] K. Nozaki and N. Bekki, Pattern selection and spatiotemporal transition to chaos in the Ginzburg-Landau equation, Phys. Rev. Letters, Vol. 51, \#24, 12 Dec. 1983

[4] Y. Kuramoto, Diffusion induced chaos in reaction systems, Supp. Prog. Theor. Phys., No. 64, 1978

[5] C. S. Bretherton and E. A. Spiegel, Intermittency through modulational instability, Physics Letters, Vol. 96A, \#3, 20 June 1983

[6] L. Keefe, Dynamics of perturbed wavetrain solutions to the Ginzburg-Landau equation, Ph.D. Dissertation, University of Southern California, 1984

[7] R. J. Deissler, Noise-sustained structure intermittency and the Ginzburg-Landau equation, submitted for publication.

[8] A. C. Newell and J. A. Whitehead, Finite bandwidth, finite amplitude convection, J. Fluid Mech. 38, 279 (1969)

[9] K. Stewartson and J. T. Stuart, $A$ non-linear instability theory for a wave system in plane Poiseuille flow, J. Fluid Mech. 48, 529 (1971)

[10] R. C. DiPrima, W. Eckhaus, and L. A. Segel, Non-linear wave-number interaction in near-critical two dimensional flows, J. Fluid Mech. 49, 705 (1971)

[11] D. J. Benney and G. J. Roskes, Wave instabilities, Stud. Appl. Math. 48, 377 (1969)

[12] A. Davey and K. Stewartson, On three-dimensional packets of surface waves, Proc. Roy. Soc. London A338, $101(1974)$

[13] T. B. Benjamin and J. E. Feir, The disintegration of wave trains on deep water, J. Fluid Mech. 27, 417 (1967)

[14] H. Hasimoto and H. Ono, Nonlinear modulation of gravity waves, J. Phys. Soc. Japan 33, 805 (1972)

[15] A. Jeffrey and T. Kawahara, Asymptotic methods in nonlinear wave theory, Pittman, 1982

[16] G. B. Whitham, Linear and nonlinear waves, John Wiley \& Sons, Inc., 1974

[17] W. Eckhaus, Studies in non-linear stability theory, Springer Tracts in Natural Philosophy, vol. 6, SpringerVerlag, 1965

[18] J. T. Stuart and R. C. DiPrima, The Eckhaus and Benjamin-Feir resonance mechanisms, Proc. Roy. Soc. Lond. A 362, 27 (1978)

[19] A. Thyagaraja, in Nonlinear waves, ed. L. Debnath, Chapter 17: "Recurrence Phenomena and the Number of Effective Degrees of Freedom in Nonlinear Wave Motions," Cambridge, 1983

[20] A. Thyagaraja, Recurrence, dimensionality, and Lagrange stability of solutions of the nonlinear Schrödinger equation. Phys. Fluids 24 (11), Nov. 1981

[ 21] V. V. Nemytiskii and V. V. Stepanov, Qualitative theory of differential equations, Princeton Univ. Press, 1960 